\title{
An example of a positive definite function which is not of positive type on $\mathrm{Z}^{2}$
}

\author{
Koji Furuta* and Nobuhisa Sakakibara**
}

Let $S=(S,+, *)$ be an abelian $*$-semigroup with the identity $0, \mathcal{H}$ a complex Hilbert space with an inner product $\langle\cdot, \cdot\rangle$ and $B(\mathcal{H})$ the set of bounded linear operators on $\mathcal{H}$. A function $\varphi: S \rightarrow B(\mathcal{H})$ is called of positive type if

$$
\sum_{i, j=1}^{n}\left\langle\varphi\left(s_{i}+s_{j}^{*}\right) \xi_{i}, \xi_{j}\right\rangle \geqq 0
$$

for all $n \geqq 1, s_{1}, s_{2}, \cdots, s_{n} \in S$ and $\xi_{1}, \xi_{2}, \cdots, \xi_{n} \in \mathcal{H}$. Moreover, $\varphi$ is called positive definite if

$$
\sum_{i, j=1}^{n} c_{i} \overline{c_{j}}\left\langle\varphi\left(\dot{s}_{i}+s_{j}^{*}\right) \xi, \xi\right\rangle \geqq 0
$$

for all $n \geqq 1, s_{1}, s_{2}, \cdots, s_{n} \in S, c_{1}, c_{2}, \cdots, c_{n} \in \mathrm{C}$ and $\xi \in \mathcal{H}$. Every function of positive type is positive definite, and every scalar-valued, positive definite function is of positive type. But a positive definite function is not necessarily of positive type. In fact, T. M. Bisgaard demonstrated that there exists an explicit example of a positive definite function which is not of positive type on $\left(\mathrm{N}_{0},+, x^{*}=x\right)$ where $\mathbf{N}_{0}:=\{0,1,2, \cdots\}$ (see [1, Theorem 1]), and we did on $\left(\mathrm{Z},+, x^{*}=x\right)$ (see [3, Theorem 3.7]). For abelian $*$-semigroups $\left(\mathrm{N}_{0}^{2},+, x^{*}=x\right)$ and $\left(\mathrm{Z}^{2},+, x^{*}=x\right)$, is there such an example? When $\left(\mathrm{N}_{0}^{2},+, x^{*}=x\right)$, the answer is clear because we have the zero extension of Bisgaard's example (i.e. $\varphi(n, 0)$ is Bisgaard's and $\varphi(n, m)=0$ for $m>0)$. In this paper, we shall show such an explicit example on $\left(\mathrm{Z}^{2},+, x^{*}=x\right)$.

REMARK. A function $\varphi: S \rightarrow B(\mathcal{H})$ is called a operator moment function if there exists a $B(\mathcal{H})_{+}$-valued measure $F$ on $S^{*}$ such that

$$
\langle\varphi(s) \xi, \eta\rangle=\int_{S^{*}} \rho(s) d\langle F(\rho) \xi, \eta\rangle \quad \text { for } s \in S, \xi, \eta \in \mathcal{H}
$$

Every operator moment function is of positive type. But a function of positive type is not necessarily an operator moment function. In fact, even a scalarvalued, positive definite function is not necessarily a moment function. So an

Received September 29, 1998.

1991 Mathematics Subject Classification. 43A35.

* Department of Mathematics, Musashi Institute of Technology, Tamazutsumi, Setagaya,

Tokyo 158-8557, Japan.

email:

** Faculty of Engineering, Ibaraki University, Nakanarusawa 4-12-1, Hitachi 316-8511, Japan. email: 
abelian *-scmigroup $S$ is called operator semiperfect (resp. semiperfect) if cvery function of positive type (resp. scalar-valued, positive definite function) on $S$ is a operator moment function (resp. moment function). Operator semiperfect *-semigroups have been analyzed by Bisgaard ([2]), Stochel and Szafraniec ([4]), and the authors $([3])$. Semiperfect $*$-semigroups have becn more analyzed by Bisgaard and the authors.

Let us first define a linear order $\ll$ on $\mathrm{Z}^{2}$ as follows:

$$
\begin{aligned}
\left(n_{1}, m_{1}\right) \ll\left(n_{2}, m_{2}\right): \Longleftrightarrow & \cdot\left|n_{1}\right|+\left|m_{1}\right|<\left|n_{2}\right|+\left|m_{2}\right| \quad \text { or } \\
& \cdot\left|n_{1}\right|+\left|m_{1}\right|=\left|n_{2}\right|+\left|m_{2}\right|, \\
& m_{1}>0, m_{2}>0, n_{1}<n_{2} \quad \text { or } \\
\cdot & \left|n_{1}\right|+\left|m_{1}\right|=\left|n_{2}\right|+\left|m_{2}\right|, m_{1}>0, m_{2} \leqq 0 \quad \text { or } \\
\cdot & \left|n_{1}\right|+\left|m_{1}\right|=\left|n_{2}\right|+\left|m_{2}\right|, m_{1} \leqq 0, m_{2} \leqq 0, n_{2}<n_{1} .
\end{aligned}
$$

Arrange points of $\mathbf{Z}^{2}$ by this order, i.e.

$$
\begin{aligned}
& x_{0}:=(0,0), \quad x_{1}:=(0,1), \quad x_{2}:=(1,0), \quad x_{3}:=(0,-1), \\
& x_{4}:=(-1,0), \quad x_{5}:=(-1,1), \quad x_{6}:=(0,2),
\end{aligned}
$$

and let $a_{n}:=2^{(n+2) !}, n \geqq 1$. The following is our theorem, in which the choices of $2 \times 2$ matrices is similar to those in Bisgaard's example.

Theorem. Let $\varphi: \mathbf{Z}^{2} \rightarrow M_{2}(\mathbf{C})$ be a function defined by

$$
\begin{array}{ll}
\varphi\left(x_{0}\right):=\left(\begin{array}{ll}
4 & 0 \\
0 & 1
\end{array}\right), & \varphi\left(x_{i}\right):=\left(\begin{array}{cc}
0 & 2 \\
2 & 0
\end{array}\right), \quad 1 \leqq i \leqq 5, \\
\varphi\left(x_{6}\right):=\left(\begin{array}{ll}
1 & 0 \\
0 & 4
\end{array}\right), & \varphi\left(x_{n}\right):=\left(\begin{array}{cc}
a_{n} & 0 \\
0 & a_{n}
\end{array}\right), \quad n \geqq 7 .
\end{array}
$$

Then $\varphi$ is positive definite and not of positive type on $\left(\mathbf{Z}^{2},+, x^{*}=x\right)$.

Proof. To see that $\varphi$ is positive definite on $\left(\mathrm{Z}^{2},+, x^{*}=x\right)$, we shall prove

$$
D_{n}(\xi):=\left|\begin{array}{ccc}
\left\langle\varphi\left(x_{0}+x_{0}\right) \xi, \xi\right\rangle & \cdots & \left\langle\varphi\left(x_{0}+x_{n}\right) \xi, \xi\right\rangle \\
\vdots & \ddots & \vdots \\
\left\langle\varphi\left(x_{n}+x_{0}\right) \xi, \xi\right\rangle & \cdots & \left\langle\varphi\left(x_{n}+x_{n}\right) \xi, \xi\right\rangle
\end{array}\right|>0
$$

for $n \geqq 0$ and $\xi=\left(\begin{array}{c}\alpha \\ \beta\end{array}\right) \in \mathbf{C}^{2}$ with $|\alpha|^{2}+|\beta|^{2}=1$. We have

$$
\begin{aligned}
D_{0}(\xi) & =4|\alpha|^{2}+|\beta|^{2} \geqq 1 \\
D_{1}(\xi) & =\left(4|\alpha|^{2}+|\beta|^{2}\right)\left(|\alpha|^{2}+4|\beta|^{2}\right)-16(\operatorname{Re}(\alpha \bar{\beta}))^{2} \\
& =4|\alpha|^{4}+4|\beta|^{4}+17|\alpha \beta|^{2}-16(\operatorname{Re}(\alpha \bar{\beta}))^{2} \\
& \geqq 4|\alpha|^{4}+4|\beta|^{4}+|\alpha|^{2}|\beta|^{2} \\
& =4-7|\alpha|^{2}|\beta|^{2} \geqq 4-\frac{7}{4}>1 .
\end{aligned}
$$


Let $n \geqq 2$ and suppose that $D_{n-1}(\xi) \geqq 1$. We show that $D_{n}(\xi) \geqq 1$. Suppose the following:

(*) For every $n \geqq 2$, there exists a natural number $m \geqq n, m \geqq 8$ such that

$$
\begin{aligned}
& \left\|\varphi\left(x_{n}+x_{n}\right)\right\|=a_{m} \\
& \left\|\varphi\left(x_{j}+x_{k}\right)\right\| \leqq a_{m-1}, \quad 0 \leqq j \leqq n, 0 \leqq k \leqq n-1
\end{aligned}
$$

Then

$$
\begin{aligned}
D_{n}(\xi)= & \left\langle\varphi\left(x_{n}+x_{n}\right) \xi, \xi\right\rangle D_{n-1}(\xi) \\
& \quad+\sum_{k=0}^{n-1}(-1)^{n+1+k+1}\left\langle\varphi\left(x_{k}+x_{n}\right) \xi, \xi\right\rangle D_{k}^{\prime}(\xi) \\
\geqq & a_{m}-n a_{m-1}^{n+1} n ! \\
\geqq & 2^{(m+2) !}-(n+1) ! 2^{(m+1) !(n+1)} \\
\geqq & 2^{(m+1) !(m+1)}\left(2^{(m+1) !}-(n+1) !\right) \\
\geqq & 2^{(m+1) !}-(m+1) ! \\
\geqq & 1
\end{aligned}
$$

where

$$
D_{k}^{\prime}(\xi):=\left|\begin{array}{ccc}
\left\langle\varphi\left(x_{0}+x_{0}\right) \xi, \xi\right\rangle & \cdots & \left\langle\varphi\left(x_{0}+x_{n-1}\right) \xi, \xi\right\rangle \\
\vdots & \ddots & \vdots \\
\left\langle\varphi\left(x_{k-1}+x_{0}\right) \xi, \xi\right\rangle & \cdots & \left\langle\varphi\left(x_{k-1}+x_{n-1}\right) \xi, \xi\right\rangle \\
\left\langle\varphi\left(x_{k+1}+x_{0}\right) \xi, \xi\right\rangle & \cdots & \left\langle\varphi\left(x_{k+1}+x_{n-1}\right) \xi, \xi\right\rangle \\
\vdots & \ddots & \vdots \\
\left\langle\varphi\left(x_{n}+x_{0}\right) \xi, \xi\right\rangle & \cdots & \left\langle\varphi\left(x_{n}+x_{n-1}\right) \xi, \xi\right\rangle
\end{array}\right|,
$$

$k=0,1,2, \cdots, n-1$. Thus, by induction, we get $D_{n}(\xi) \geqq 1$ for $n \geqq 0$. Suppose that

(**) $\quad$ for every $n \geqq 2, \quad x_{n}+x_{k} \ll x_{n}+x_{n}, \quad k=0,1,2, \cdots, n-1$.

Then we can easily prove $(*)$. Therefore let us prove $(* *)$.

Put $x_{n}=:(p, q)$ and $x_{k}=:(r, s)$. Then $x_{n}+x_{n}=(2 p, 2 q)$ and $x_{n}+x_{k}=$ $(p+r, q+s)$. In case that $|r|+|s|<|p|+|q|$ or $p r<0$ or $q s<0$, it is easily seen that $|p+r|+|q+s|<|2 p|+|2 q|$. Hence $x_{n}+x_{k} \ll x_{n}+x_{n}$. Suppose that $|r|+|s|=|p|+|q|, p r \geqq 0$ and $q s \geqq 0$. When $p>0$ and $q \geqq 0$, the condition $x_{k} \ll x_{n}$ implies $p>r \geqq 0$ and $s \geqq 0$. Hence $(p+r)+(\bar{q}+s)=2 p+2 q$, $0 \leqq 2 q<q+s$ and $p+r<2 p$. Therefore $x_{n}+x_{k} \ll x_{n}+x_{n}$. When $p \geqq 0$ and $q<0$, we have $r>p \geqq 0$ and $0 \geqq s>q$. Hence $(p+r)+|q+s|=2 p+|2 q|$, $2 q<q+s<0$ and $p+r>2 p$. Therefore $x_{n}+x_{k} \ll x_{n}+x_{n}$. When $p \leqq 0$ 
and $q>0$, since $0 \geqq p>r$ and $q>s>0$, we have $|p+r|+(q+s)=|2 p|+2 q$, $0<q+s<2 q$ and $p+r<2 p$. Therefore $x_{n}+x_{k} \ll x_{n}+x_{n}$. When $p<0$ and $q \leqq 0$, since $0 \geqq r>p$ and $s<q \leqq 0$, we have $|p+r|+|q+s|=|2 p|+|2 q|$, $q+s<2 q \leqq 0$ and $2 p<p+r$. Therefore $x_{n}+x_{k} \ll x_{n}+x_{n}$. We finished the proof of $(* *)$.

$$
\begin{aligned}
& \text { Put } \xi_{0}:=\left(\begin{array}{l}
0 \\
1
\end{array}\right) \text { and } \xi_{1}:=\left(\begin{array}{c}
-1 \\
0
\end{array}\right) \text {. Then } \\
& \qquad \sum_{i, j=0}^{1}\left\langle\varphi\left(x_{i}+x_{j}\right) \xi_{i}, \xi_{j}\right\rangle=-2<0,
\end{aligned}
$$

which implies that $\varphi$ is not of positive type. This completes the proof.

ACKNOWLEDGEMENTS. We would like to thank the referee for offering many helpful suggestions. This work of the second author was partly supported by the Saneyoshi Scholarship Foundation.

\section{References}

[1] T. M. Bisgaard, Positive definite operator sequences, Proc. Amer. Math. Soc. 121 (1994), 1185-1191.

[2] T. M. Bisgaard, Extensions of Hamburger's theorem, Semigroup Forum 57 (1998), 397429.

[3] K. Furuta and N. Sakakibara, Operator moment problems on abelian *-semigroups, Math. Japon. (to appear).

[4] J. Stochel and F. H. Szafraniec, The complex moment problem and subnormality : A polar decomposition approach, J. Funct. Anal. 159 (1998), 432-491. 